\title{
ASSESSMENT OF GENOTOXICITY OF VINCRISTINE, VINBLASTINE AND VINORELBINE IN HUMAN CULTURED LYMPHOCYTES: A COMPARATIVE STUDY
}

Mhaidat $\mathrm{NM}^{1, *}$, Alzoubi $\mathrm{KH}^{1}$, Khabour $\mathrm{OF}^{3}$, Alawneh $\mathrm{KZ}^{2}$, Raffee $\mathrm{LA}^{2}$, Alsatari $\mathrm{ES}^{4}$, Hussein $\mathrm{EI}^{5}$, Bani-Hani $\mathrm{KE}^{6}$

*Corresponding Author: Nizar M. Mhaidat, Ph.D., Department of Clinical Pharmacy, Faculty of Pharmacy, Jordan University of Science and Technology, Amman-Ramtha Hwy, Irbid 22110, Jordan. Tel: +9622-720-100. Fax:+962-2-720-1075. E-mail: nizarm@just.edu.jo

\begin{abstract}
Vincristine (VCR), vinblastine (VBL) and vinorelbine (VRL) are anticancer agents from the Vinca alkaloid family that have the potential to induce genotoxic effect. The aim of the present study was to compare the genotoxic effect of VCR, VBL and VRL. Levels of 8-hydroxy-2-deoxy guanosine (8-OHdG) and sister chromatid exchanges (SCEs) were measured in cultured human blood lymphocytes treated with VCR, VBL and VRL at concentrations of 0.01 and $0.1 \mu \mathrm{g} / \mathrm{mL}$. Results showed that VCR, VBL and VRL significantly increased the $8-\mathrm{OHdG}$ levels $(p<0.05)$, whereas it did not cause a significant increase in the frequencies of SCEs in human blood lymphocytes as compared to controls. On the other hand, all three agents significantly increased cells mitotic index $(p<0.05)$. At both tested concen-
\end{abstract}

\footnotetext{
${ }^{1}$ Department of Clinical Pharmacy, Faculty of Pharmacy, Jordan University of Science and Technology, Irbid, Jordan

${ }^{2}$ Faculty of Medicine, Jordan University of Science and Technology, Irbid, Jordan

${ }^{3}$ Department of Medical Laboratory Sciences, Faculty of Applied Medical sciences, Jordan University of Science and Technology, Irbid, Jordan

${ }^{4}$ Department of Applied Biological Sciences, Faculty of Science and Arts, Jordan University of Science and Technology, Irbid, Jordan

${ }^{5}$ Department of Biology, Faculty of Science, Yarmouk University, Irbid, Jordan

${ }^{6}$ Faculty of Medicine, Hashemite University, Zarqaa, Jordan
}

trations, the magnitude of the increase in $8-\mathrm{OHdG}$ was $\mathrm{VBL}>\mathrm{VCR}>\mathrm{VRL}$. In conclusion, VCR, VBL and VRL induce DNA damage as indicated by the increase in the 8-OHdG biomarker but with different magnitude.

Keywords: Chromosomal aberrations; Human blood lymphocyte; 8-hydroxy-2-deoxy guanosine (8-OHdG); Sister chromatid exchanges; Vincristine (VCR); Vinblastine (VBL); Vinorelbine (VRL).

\section{INTRODUCTION}

Vinca alkaloids are a subset of drugs derived from the Madagascar periwinkle plant (Catharanthus roseus) [1]. They include the natural products vincristine (VCR) and vinblastine (VBL) and the semi-synthetic derivative vin-orelbine (VRL). Vinca alkaloids have been used for cancer management [2]. Chemically, vinca alkaloids have dimeric chemical structures composed of two basic multi-ringed units, an indole nucleus (catharanthine) and a dihydroindole nucleus (vindoline), joined together with other complex systems $[2,3]$.

Different Vinca alkaloids have their own unique properties. The VBL inhibits angiogenesis [4]. It is also associated with anti-diuretic hormone secretion and angina, and applied to treat Hodgkin's disease, non-Hodgkin's lymphoma and breast cancer [5]. The VRL showed a significant anti-tumor activity in patients with breast cancer and induces anti-proliferative activity in osteosarcoma [6]. Moreover, VCR has been shown to have a mild myelo-suppressive action 
$[7,8]$. It is also widely used to treat pediatric leukemias, solid tumors, and hematological malignancies [2]. During cell division, Vinca alkaloids bind to the building blocks of a protein called tubulin, inhibiting its formation, which normally works in cells to create mitotic spindle [9].

Previous studies have shown that Vinca alkaloids have the potential to induce genotoxic effects in different biological systems. The VCR and VBL have been shown to increase the frequency of micronuclei in experimental animals and in cultured human lymphocytes [10-13]. In addition, they have also been shown to cause chromosomal mutations in vivo and in cultured cancer cells $[14,15]$. In cultured human lymphocytes, VRB and VCR increased the rate of micronucleus formation [16]. In Drosophila, VCR and VBL induced a significant genotoxic effect as measured using wing somatic mutation and the recombination test [17]. However, some other studies have shown lack of mutagenic effect for Vinca alkaloids in vivo and in cultured cells [18-20]. Thus, the genotoxicity of Vinca alkaloids is still controversial. In addition, oxidative DNA damage induced by these compounds has still not been investigated. The aim of the present study was to compare the genotoxic effect of VCR, VBL and VRL on human cultured lymphocytes using 8-hydroxy-2-deoxy guanosine (8-OHdG) and sister chromatid exchanges (SCEs) assays. The 8-OHdG is a marker that reflects oxidative DNA damage, while the SCEs assay reflects genotoxicity induced as a result of breaks in DNA during DNA recombination.

\section{MATERIALS AND METHODS}

Subjects. Five healthy male nonsmoking volunteers, with an age range of 20-25 years old, were the blood donors. Exclusion criteria were alcohol, cigarette smoking, medications and vitamin use. A sample of whole venous blood $(15 \mathrm{~mL})$ was collected in heparin tubes from each donor under sterile conditions. Whole blood cells were cultured within 1 hour of sampling. Informed consent was obtained from each volunteer. This study was approved by the Institutional Review Board of Jordan University of Science and Technology, Irbid, Jordan.

Drugs and Treatment. Vincristine, VBL and VRL were purchased from Sigma-Aldrich Produktions GmbH (Steinheim am Albuch, Germany).
To evaluate the effect of VCR, VBL and VRL on DNA, seven groups were used: a control group and drug-treated groups (VCR, VBL and VRL; each at concentrations of 0.01 and $0.1 \mu \mathrm{g} / \mathrm{mL}$ ). The control group was treated with distilled water. Drug-treated groups were treated with the corresponding drug 4 hours prior to harvesting. This was based on previous studies [21,22].

Lymphocytes Culture. Blood cultures were set up by inoculating $1 \mathrm{~mL}$ of freshly drawn blood into 75 $\mathrm{mL}$ tissue-culture flasks containing $9 \mathrm{~mL}$ of peripheral blood (PB)-Max medium composed of Roswell Park Memorial Institute (RPMI) 1640 medium with $15.0 \%$ fetal bovine serum (FBS), $1.0 \%$ penicillinstreptomycin and $3.0 \%$ of phytohaemagglutinin (Gibco-Invitrogen Ltd., Paisley, Ren-frewshire, UK). To collect metaphase cells, cultures were exposed to 100 $\mu \mathrm{L}$ of $10 \mu \mathrm{g} / \mathrm{mL}$ colcemid (Gibco-Invitrogen Ltd.) 2 hours prior to cell harvesting [23,24].

The 8-Hydroxy-2-Deoxy Guanosine Assay. The $8-\mathrm{OHdG}$ assay was performed as previously described [25]. In brief, blood cultures were set up by inoculating $0.5 \mathrm{~mL}$ of freshly drawn blood into $50 \mathrm{~mL}$ culture flasks containing $4.5 \mathrm{~mL}$ of PB-Max medium. Then, the cultures were incubated for 72 hours at $37^{\circ} \mathrm{C}$. Treatment was as described above. Cultures were then centrifuged at $1000 \times \mathrm{g}$ and 200 $\mu \mathrm{L}$ from each was used for the $8-\mathrm{OHdG}$ assay. Competitive enzyme-linked-immunosorbent serologic assay (ELISA) for 8-OHdG were performed according to the manufacturer's protocol protocol (Abcam Inc., Cambridge, MA, USA). Plates were read at $405 \mathrm{~nm}$ using an Epoch Biotek microplate reader (BioTek, Winooski, VT, USA). Levels of 8-OHdG were calculated from the blotted standard curve.

Sister Chromatid Exchange Assay. A $25 \mu \mathrm{L}$ of $0.01 \mathrm{~g} / \mathrm{L} \mathrm{mL}$ bromodeoxyridine (BrdU; SigmaAldrich, St. Louis, MO, USA), was added to the culture media prior to incubation and throughout the experiment $[23,24]$. All cultures were maintained in total darkness to minimize photolysis of BrdU [26-28]. The culture initiation and slide preparation were similar to that described for the chromosomal aberration assay. Air-dried slides were differentially stained by $10 \mu \mathrm{g} / \mathrm{mL}$ of Hoechst 32285 dye solution (Fluka, Münich, Germany) for 15 min., followed by rinsing in water and mounted in McILvian buffer (Fisher Scientific, Waltham, MA, USA) (pH 8.0). The slides were then irradiated with two UV lamps 
(350 $\mathrm{nm}$ and $15 \mathrm{~W}$ each) at a distance of $7 \mathrm{~cm}$ for $35 \mathrm{~min}$. at $40{ }^{\circ} \mathrm{C}[22]$. Slides were then rinsed with distilled water, restained for 6-8 min. with $5.0 \%$ Giemsa in phosphate buffer ( $\mathrm{pH} 6.8$ ) and then air-dried $[27,29,30]$. Sister chromatid exchanges were scored using second division cells (M2, 50 cells per donor) that contained 42-46 chromosomes and high resolution (Nikon, Shinagawa, Japan) light microscopy $[25,31]$. Cells in M2 phase have two differentially stained chromatids, one lightly stained and one darkly stained, while M1 phase cells are uniformly stained (two chromatids are darkly stained), and M3 phase cells contain a mixture of lightly stained, darkly stained and differentially stained chromatids [26].

Cell Kinetics Analysis. The mitotic index was calculated by analyzing at least 1000 cells from each subject and scoring the cells that were in metaphase as previously described [26]. For the cell proliferation index, 100 metaphase cells from each donor were scored. The proliferation index was calculated using the following formula: $(1 \mathrm{X} \mathrm{M} 1+2 \mathrm{X} \mathrm{M} 2+3$ $X \geq M 3) / 100$, where $M 1, M 2$ and $M 3$ are the number of cells at the first, second and third metaphase, respectively [24,32]. Depending on the proliferation index, the average generation time was calculated as the number of hours for the cells in BrdU (SigmaAldrich), divided by the proliferation index [28].

Statistical Analyses. Statistical analysis was performed using Graphpad Prism statistical software version 4 (GraphPad Software, Inc., La Jolla, CA, USA). Data were expressed as mean \pm standard error
(SE). The comparisons of parameters were performed using one-way analysis of variance (ANOVA) followed by Tukey's multiple comparison test. Differences were regarded as significant at $p<0.05$.

\section{RESULTS}

Figure 1 shows the level of $8-\mathrm{OHdG}$ in all groups. Levels of 8-OHdG in drug-treated groups (VCR, VBL and VRL; each at concentrations of 0.01 and $0.1 \mu \mathrm{g} / \mathrm{mL}$ ) were higher than those in the control group $(p<0.05)$. These results indicate that VCR, VBL and VRL at concentrations of 0.01 and $0.1 \mu \mathrm{g} /$ $\mathrm{mL}$ induce oxidative DNA damage. Figure 1 also shows that at both tested concentrations, the magnitude of the increase in $8-\mathrm{OHdG}$ was higher in the VBL-treated group compared to the VCR and VRL groups (Figure 1).

Sister chromatid exchanges were observed in differentially stained M2 metaphase cells that have 42-48 chromosomes. There was an increase in SCEs frequency between control and drug-treated groups (VCR, VBL and VRL, each at concentrations of 0.01 and $0.1 \mu \mathrm{g} / \mathrm{mL}$ ). However, this increase did not reach a significant level $(p>0.05)$ (Figure 2).

Previous studies showed that Vinca alkaloid drugs affect spindle fibers and causes cell arrest in the mitotic phase. As expected, the mitotic index was higher in the VCR-, VBL- and VRL-treated groups compared to the control group (Figure 3 ). In addition, in all treated groups, the effect was dose dependent.

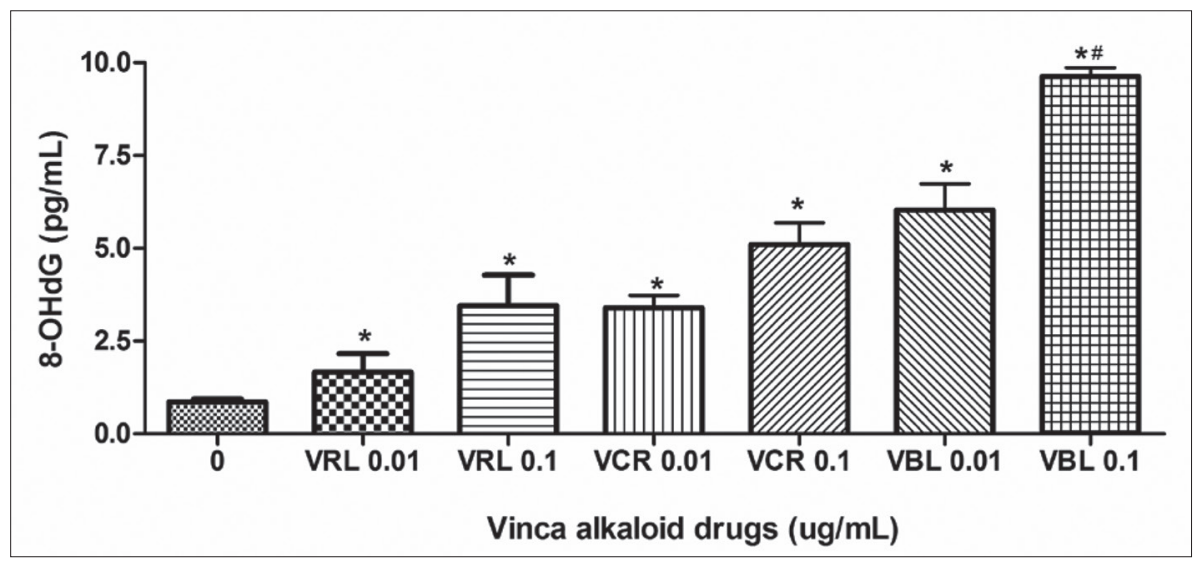

Figure 1. The level of 8-OHdG values in controls, VCR, VBL and VRL groups; each at concentrations of 0.01 and $0.1 \mu \mathrm{g} / \mathrm{mL}$. The levels of $8-\mathrm{OHdG}$ in all drug-treated groups at concentrations of 0.01 and $0.1 \mu \mathrm{g} / \mathrm{mL}$ were higher than control group. The asterisks $(*)$ indicate significant differences $(p<0.05)$ from the control group. The hash $(\#)$ indicates significant difference from all other groups. 


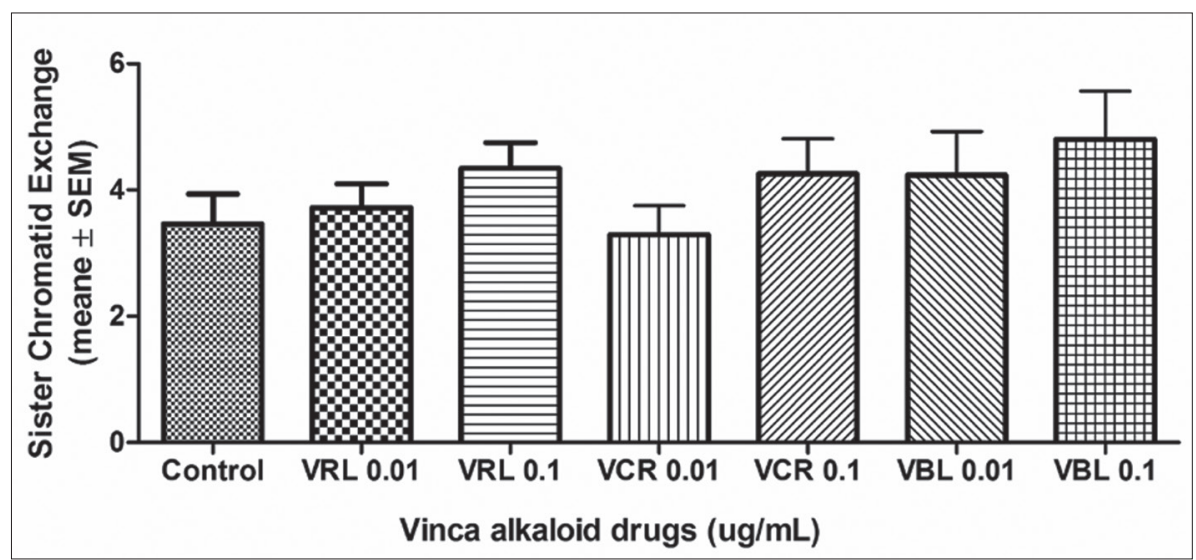

Figure 2. The average of SCEs/cell in controls, VCR, VBL and VRL groups; each at concentrations of 0.01 and $0.1 \mu \mathrm{g} / \mathrm{mL}$ in cultured blood cells. Increases were detected among the groups. However, it did not reach significant levels (control vs. VCR group: $p>0.05$; control vs. VBL group: $p>0.05$, and control $v s$. VRL group: $p>0.05$ ).

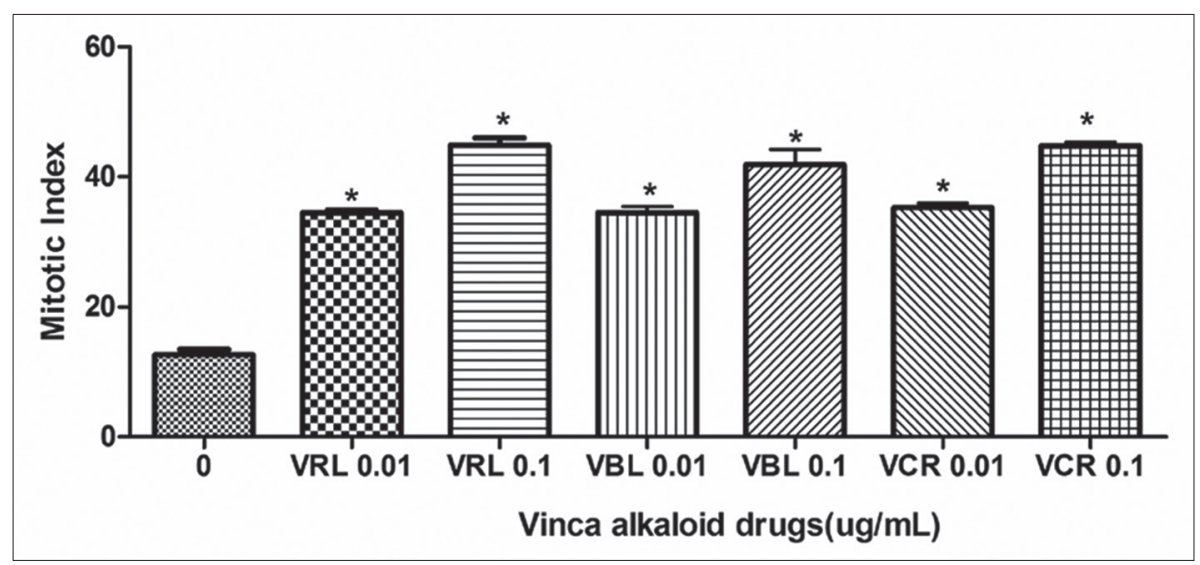

Figure 3. The mitotic index in cultured cells treated with VCR, VBL and VRL; each at concentrations of 0.01 and $0.1 \mu \mathrm{g} / \mathrm{mL}$. The value of the mitotic index was significantly higher $(p<0.05)$ in lymphocytes treated with VCR, VBL and VRL; each at concentrations of 0.01 and $0.1 \mu \mathrm{g} / \mathrm{mL}$.

Moreover, the magnitude of mitotic arrest was similar in VCR, VBL and VRL (Figure 3).

\section{DISCUSSION}

The current study indicated that VCR, VBL and VRL, at concentrations of 0.01 and $0.1 \mu \mathrm{g} / \mathrm{mL}$, induced oxidative DNA damage as shown using 8-OHdG biomarker. The magnitude of the increase in 8-OHdG was higher in cells treated with VBL as compared to VCR and VRL.

The VCR, VBL and VRL have been reported to induce reactive oxygen and nitrogen species. For example, VRL has been shown to cause oxidative injury to cultured human endothelial cells [33]. In addition, treatment of human bronchial epithelial cell with VCR was associated with oxidative DNA damage as measured using normal alkaline and formamidopyrimidine-DNA-glycosylase (FPG) modified comet assays $[10,33,34]$. It was recently reported that chronic treatment with antioxidant agents prevented che-motherapies, including VBL, induced oxidative damage and restores normal mitochondrial function in hepatic cells [35]. In this study, a significant increase in the level of $8-\mathrm{OHdG}$, which is a standard biomarker for oxidative DNA damage [36,37], was shown when cultured human lymphocytes were treated with VCR, VBL and VRL. This 
variation could be due to the unique properties of these Vinca alkaloids (see Introduction). However, this point requires further investigation.

Current results show that at both tested doses, VBL induced increases in the levels of 8-OHdG that were larger in magnitude than those induced by both VCR and VRL. The exact mechanism for such amplified effect of VBL on the levels of 8-OHdG is unknown. However, previous studies have shown a differential effect for VBL on bone marrow cell as compared to fetal liver cells [17]. Additionally, VBL was shown to alter calcium homeostasis via mitochondrial membranes leading to substantiated cyto-toxicity as compared to VCR [38].

Results of this study showed no significant increase in SCEs frequency in the VCR-, VBL- and VRL-treated groups. Previous studies indicated the possible genotoxicity of VCR and VBL [12]. For example, VCR was shown to induce DNA misrepair, telomere end fusions, nuclear buds, and increased frequency of gene mutations, all of which points to the possible genotoxicity of VCR [10]. Vincristine was also shown to increase the number of micronucleated cells, indicating its possible genotoxcicity $[39,40]$. All three agents, VCR, VBL and VRL, showed signs of genotoxicity in the Drosophila model [17]. On the other hand, VBL was recently shown to induce chromosomal aberrations and increase mitotic index in vitro in bone marrow cells, which was prevented by pretreatment with various doses of caffeine [41]. However, using the mouse lymphoma assay, VCR pretreatment for 3 hours did not show positive results for genotoxicity [42]. Additionally, increasing doses of VCR or VBL treatments were found to decrease the numbers of chromosomal aberrations along with an increase in number of micronucleated cells $[11,43]$. In another in vivo study in pregnant mice, a 2-week treatment with VCR did not induce an increase in SCEs in both maternal and fetal tissues, although it was capable of increasing the frequency of micronuclei [44]. Thus, it seems that Vinca alkaloids are able to induce certain types of DNA alterations such as oxidative and micronuclei but not others (i.e., SCEs). This could be due to their mechanism of action that is associated with activation of apoptosis through interference with spindle fiber formation and mitochondrial function. Moreover, the aberrant variations in the genotoxicity results for VCR in in vitro cultured cell techniques and mammalian models could be related to variation in models, doses and duration of drug treatment.

The current results showed increased mitotic index in cells treated with VCR, VBL and VRL, indicating an antimitotic activity of these compounds. This is consistent with previous results showing that VCR increased apoptotic cell numbers and ratios and decreased the nuclear division in a dose-dependent manner, thus, showing the cytotoxicity of VCR $[10,40]$. In fact, Vinca alkaloids including VCR, VBL and VRL, are known to exert their cytoxicity via arresting mitosis and going into interphase $[45,46]$, which is consistent with the current results. Based on the these results, we concluded that VCR, VBL and VRL induce oxidative DNA damage in whole blood lymphocytes.

Declaration of Interest. This project was supported by a grant from the Deanship of Research at Jordan University of Science and Technology. The authors report no conflicts of interest. The authors alone are responsible for the content and writing of this article.

\section{REFERENCES}

1. Facchini PJ, De Luca V. Opium poppy and Madagascar periwinkle: Model non-model systems to investigate alkaloid biosynthesis in plants. Plant J. 2008; 54(4): 763-784.

2. Moudi MR, Go R, Yien CY, Nazre M. Vinca alkaloids. Int J Prev Med. 2013; 4(11): 1231-1235.

3. Duffin J. Poisoning the spindle: Serendipity and discovery of the anti-tumor properties of the Vinca alkaloids. Pharm Hist. 2002; 44(2?): 64-76.

4. Ribatti D, Guidolin D, Conconi MT, Nico B, Baiguera S, Parnigotto PP, et al. Vinblastine inhibits the angiogenic response induced by adrenomedullin in vitro and in vivo. Oncogene. 2003; 22(41): 6458-6461.

5. Toussaint C, Izzo J, Spielmann M, Merle S, MayLevin F, Armand JP, et al. Phase I/II trial of continuous infusion vinorelbine for advanced breast cancer. J Clin Oncol. 1994; 12(10): 2102-2112.

6. Freyer G, Delozier T, Lichinister M, Gedouin D, Bougnoux P, His P, et al. Phase II study of oral vinorelbine in first-line advanced breast cancer chemotherapy. J Clin Oncol. 2003; 21(1): 35-40. 
7. Rusthoven J, Bramwell V, Stephenson B. Use of granulocyte colony-stimulating factor (G-CSF) in patients receiving myelosuppressive chemotherapy for the treatment of cancer. Provincial Systemic Treatment Disease Site Group. Cancer Prev Control. 1998; 2(4): 179-190.

8. Hirano M, Okamoto M, Maruyama F, Ezaki K, Shimizu K, Ino T, et al. Alternating non-crossresistant chemotherapy for non-Hodgkin's lymphoma of inter-mediate-grade and high-grade malignancy. A pilot study. Cancer. 1992; 69(3): 772-777.

9. Coderch C, Morreale A, Gago F. Tubulin-based structure-affinity relationships for antimitotic Vinca alkaloids. Anticancer Agents Med Chem. 2012; 12(3): 219-225.

10. Jiang W, Lu Y, Chen Z, Chen S, Zhang M, Jin $\mathrm{L}$, et al. Studying the genotoxicity of vincristine on human lymphocytes using comet assay, micronucleus assay and TCR gene mutation test in vitro. Toxicology. 2008; 252(1-3): 113-117.

11. Choudhury RC, Palo AK, Padhy A. Cytogenetic consequences of vinblastine treatment in mouse bone marrow. Chemotherapy. 2004; 50(4): 171-177.

12. Morales-Ramirez P, Vallarino-Kelly T, CruzVallejo V. Kinetics of micronucleus induction and cytotoxicity caused by distinct antineoplastics and alkylating agents in vivo. Toxicol Lett. 2014; 224(3): 319-325.

13. Morales-Ramirez P, Vallarino-Kelly T, CruzVallejo V. Kinetics of micronucleated polychromatic erythrocyte (MN-PCE) induction in vivo by aneuploidogens. Mutat Res. 2004; 565(1): 79-87.

14. Yamada T, Odawara K, Kaneko H. Concurrent detection of gene mutations and chromosome aberrations induced by five chemicals in a CHL/ IU cell line incorporating a gpt shuttle vector. Mutat Res. 2000; 471(1-2): 29-36.

15. Arni P, Hertner T. Chromosomal aberrations in vitro induced by aneugens. Mutat Res. 1997; 379(1): 83-93.

16. Gonzalez-Cid M, Larripa I, Slavutsky I. Vinorelbine: Cell cycle kinetics and differential sensitivity of human lymphocyte subpopulations. Toxicol Lett. 1997; 93(2-3): 171-176.

17. Tiburi M, Reguly ML, Schwartsmann G, Cunha KS, Lehmann M, Rodrigues de Andrade HH.
Comparative genotoxic effect of vincristine, vinblastine, and vinorelbine in somatic cells of Drosophila melanogaster. Mutat Res. 2002; 519(1-2): 141-149.

18. Gundy S, Baki M, Bodrogi I. Vinblastine, cisplatin and bleomycin (VPB) adjuvant therapy does not induce dose-dependent damage in human chromosomes. Neoplasma. 1989; 36(4): 457-464.

19. Morgan WF, Crossen PE. A comparison of induced sister chromatid exchange levels in Chinese hamster ovary cells and cultured human lymphocytes. Environ Mutagen. 1982; 4(1): 65-71.

20. Gonzalez-Cid M, Cuello MT, Larripa I. Mitotic arrest and anaphase aberrations induced by vinorelbine in hamster cells in vitro. Anticancer Drugs. 1997; 8(5): 529-532.

21. Alzoubi K, Khabour O, Khader M, Mhaidat N, Al-Azzam S. Evaluation of vitamin B12 effects on DNA damage induced by paclitaxel. Drug Chem Toxicol. 2014; 37(3): 276-280.

22. Alzoubi K, Khabour O, Hussain N, Al-Azzam S, Mhaidat M. Evaluation of vitamin B12 effects on DNA damage induced by pioglitazone. Mutat Res. 2012; 748(1-2): 48-51.

23. Alsatari ES, Azab M, Khabour OF, Alzoubi KH, Sadiq MF. Assessment of DNA damage using chromosomal aberrations assay in lymphocytes of waterpipe smokers. Int J Occup Med Environ Health. 2012; 25(3): 218-224.

24. Khabour OF, Alsatari ES, Azab M, Alzoubi KH, Sadiq MF. Assessment of genotoxicity of waterpipe and cigarette smoking in lymphocytes using the sister-chromatid exchange assay: A comparative study. Environ Mol Mutagen. 2011; 52(3): 224-228.

25. Alzoubi KH, Khabour OF, Jaber AG, Al-Azzam SI, Mhaidat NM, Masadeh MM. Tempol prevents geno-toxicity induced by vorinostat: role of oxidative DNA damage. Cytotechnology. 2014; 66(3): 449-455.

26. Khabour OF, Alzoubi KH, Mfady DS, Alasseiri M, Hasheesh TF. Tempol protects human lymphocytes from genotoxicity induced by cisplatin. Int J Clin Exp Med. 2014; 7(4): 982-988.

27. Al-Sweedan SA, Khabour O, Isam R. Genotoxicity assessment in patients with thalassemia minor. Mutat Res. 2012; 744(2): 167-171. 
28. Kaya FF, Topaktas M. Genotoxic effects of potassium bromate on human peripheral lymphocytes in vitro. Mutat Res .2007; 626(1-2): 48-52.

29. M'Bemba-Meka P, Lemieux N, Chakrabarti SK. Role of oxidative stress and intracellular calcium in nickel carbonate hydroxide-induced sisterchromatid exchange, and alterations in replication index and mitotic index in cultured human peripheral blood lymphocytes. Arch Toxicol. 2007; 81(2): 89-99.

30. Rooney DE, Ed. Human Cytogenetics. A Practical Approach, 3rd ed., Vol. 2: Malignancy and Acquired Abnormalities. Oxford, UK: Oxford University Press, 2001.

31. Khabour OF, Saleh N, Alzoubi KH, Hisaindee S, Al-Fyad D, Al-Kaabi L, et al. Genotoxicity of structurally related copper and zinc containing Schiff base complexes. Drug Chem Toxicol. 2013; 36(4): 435-442.

32. Ivett JL, Tice RR. Average generation time: A new method of analysis and quantitation of cellular proliferation kinetics. Environ Mutagen. 1982; 4: 358-370.

33. Tsai KL, Chiu TH, Tsai MH, Chen HY, Ou HC. Vinorelbine-induced oxidative injury in human endothelial cells mediated by AMPK/PKC/ NADPH/NF-kappaB pathways. Cell Biochem Biophys. 2012; 62(3): 467-479.

34. Zhao J, Li H, Zhai Q, Qiu Y, Niu Y, Dai Y, et al. [Endonuclease modified comet assay for oxidative DNA damage induced by detection of genetic toxicants]. Zhonghua Yu Fang Yi Xue Za Zhi. 2014; 48(3): 208-212.

35. Madhu P, Reddy KP, Reddy PS. Melatonin reduces oxidative stress and restores mitochondrial function in the liver of rats exposed to chemotherapeutics. J Exp Zool A Ecol Genet Physiol. 2015; 323(5): 301-308.

36. Lee-Chen SF, Chen CL, Ho LY, Hsu PC, Chang JT, Sun CM, et al. Role of oxidative DNA damage in hydroxychavicol-induced genotoxicity. Mutagenesis. 1996; 11(5): 519-523.

37. Ogura H, Takeuchi T, Morimoto K. A comparison of the 8-hydroxydeoxyguanosine, chromo- some aberrations and micronucleus techniques for the assessment of the genotoxicity of mercury compounds in human blood lymphocytes. Mutat Res. 1996; 340(2-3): 175-182.

38. Tari C, Fournier N, Briand C, Ducet G, Crevat $A$. Action of vinca alkaloides on calcium movements through mitochondrial membrane. Pharmacol Res Commun. 1986; 18(6): 519-528.

39. Sanchez P, Llorente MT, Castano A. Flow cytometric detection of micronuclei and cell cycle alterations in fish-derived cells after exposure to three model genotoxic agents: Mitomycin C, vincristine sulfate and benzo(a) pyrene. Mutat Res. 2000; 465(1-2): 113-122.

40. Le Fevre AC, Boitier E, Marchandeau JP, Sarasin A, Thybaud V. Characterization of DNA reactive and non-DNA reactive anticancer drugs by gene expression profiling. Mutat Res. 2007; 619(1-2): 16-29.

41. Geriyol P, Basavanneppa HP, Dhananjaya BL. Protecting effect of caffeine against vinblastine (an anticancer drug) induced genotoxicity in mice. Drug Chem Toxicol. 2015; 38(2): 188-195.

42. Wang YJ, Zhang LS. [Evaluation of the genotoxicity of vincristine and colchicine using mouse lymphoma tk mutation assay]. Wei Sheng Yan Jiu. 2006; 35(2): 179-181.

43. Choudhury RC, Das B, Misra S, Jagdale MB. Cytogenetic toxicity of vincristine. J Environ Pathol Toxicol Oncol. 2000; 19(4): 347-355.

44. Xing SG, Shi X, Wu ZL, Chen JK, Wallace W, Whong WZ, et al. Transplacental genotoxicity of triethyl-enemelamine, benzene, and vinblastine in mice. Teratog Carcinog Mutagen. 1992; 12(5): 223-230.

45. Novichkova EA, Onishchenko GE, Shtil AA. Microtubule depolymerization by vincristine causes cell death after transition from $\mathrm{C}$ mitosis to interphase. Dokl Biol Sci. 2003; 393: 575-578.

46. Horton JK, Houghton PJ, Houghton JA. Relationships between tumor responsiveness, vincristine pharmacokinetics and arrest of mitosis in human tumor xenografts. Biochem Pharmacol. 1988; 37(20): 3995-4000. 
